\title{
He had it Comin': ERPs Reveal a Facilitation for the Processing of Misfortunes to Antisocial Characters
}

\author{
Pablo Rodríguez-Gómez ${ }^{1}$ - Manuel Martín-Loeches ${ }^{2,3} \cdot$ Fernando Colmenares $^{3}$ • María Verónica Romero Ferreiro ${ }^{1}$. \\ Eva M. Moreno ${ }^{1}$
}

Published online: 11 February 2020

(C) The Psychonomic Society, Inc. 2020

\begin{abstract}
Human sociality and prosociality rely on social and moral feelings of empathy, compassion, envy, schadenfreude, as well as on the preference for prosocial over antisocial others. We examined the neural underpinnings of the processing of lexical input designed to tap into these type of social feelings. Brainwave responses from 20 participants were measured as they read sentences comprising a randomly delivered ending outcome (fortunate or unfortunate) to social agents previously profiled as prosocial or antisocial individuals. Fortunate outcomes delivered to prosocial and antisocial agents aimed to tap into empathy and envy/ annoying feelings, respectively, whereas unfortunate ones into compassion for prosocial agents and schadenfreude for antisocial ones. ERP modulations in early attention-capture (100-200 ms), semantic fit (400 ms), and late reanalysis processes $(600 \mathrm{~ms})$ were analyzed. According to the functional interpretation of each of these event-related electrophysiological effects, we conclude that: 1) a higher capture of attention is initially obtained in response to any type of outcome delivered to a prosocial versus an antisocial agent (frontal P2); 2) a facilitated semantic processing occurs for unfortunate outcomes delivered to antisocial agents (N400); and 3) regardless of the protagonist's social profile, an increased later reevaluation for overall unfortunate versus fortunate outcomes takes place (Late Positive Potential). Thus, neural online measures capture a stepwise unfolding impact of social factors during language comprehension, which include a facilitated processing of misfortunes when they happen to occur to antisocial peers (i.e., schadenfreude).
\end{abstract}

Keywords Event-related potentials $\cdot$ Language processing $\cdot$ Schadenfreude $\cdot$ N400 $\cdot$ Prosociality

\section{Introduction}

Although cooperation is a widespread phenomenon in the animal kingdom, which speaks to its adaptive value, human sociality relative to other species' one is unique in various ways (Melis \& Semmann, 2010; Silk \& House, 2016; Tomasello, 2019). For example, humans are aware that their prosocial (or antisocial) actions will be evaluated positively (praised) or negatively (censured) by third parties. They also

Eva M. Moreno

emmoreno@ucm.es

1 Human Brain Mapping Unit at Instituto Pluridisciplinar, Universidad Complutense de Madrid, Paseo Juan XXIII 1, 28040 Madrid, Spain

2 Center for Human Evolution and Behavior, UCM-ISCIII, Avd/ Monforte de Lemos, 5, Madrid 28029, Spain

3 Department of Psychobiology and Methods in Behavioral Sciences, Universidad Complutense de Madrid, Campus de Somosaguas, Madrid 28223, Spain are likely to be concerned with and to actively engage in monitoring their fellow group members' compliance with group norms and in enforcing them when broken (Schmidt \& Rakoczy, 2019). It thus appears that we humans have evolved psychological and brain mechanisms for judging the moral admissibility of our own behaviour and that of our group fellows. On the basis of their behaviour, individuals will be regarded by third parties as morally deserving or undeserving (Malti et al., 2016). The outgrowth of cooperation-based sociality into morality has several far-reaching implications. For example, individuals believe that norm transgressors, uncooperative, or antisocial others deserve to be punished (Fehr \& Schurtenberger, 2018).

\section{Social Emotions}

Social and moral emotions have been categorized in several ways. For example, Jankowski and Takashi (Jankowski \& Takahashi, 2014) distinguished between self-conscious 
emotions, linked to compliance with versus transgression of group norms, such as embarrassment, guilt, shame, and pride, and fortune-of-other emotions, elicited by social comparison to others' attributes possessions or outcomes. Prototypical components of the latter are envy (i.e., displeasure at another's happiness) and schadenfreude (a term borrowed from German, indicating the pleasure, joy, or self-satisfaction that comes from learning of or witnessing the troubles, failures, or humiliation of another) (Shamay-Tsoory, AhronbergKirschenbaum, \& Bauminger-Zviely, 2014). Other social feelings include sympathy or compassion (i.e., displeasure at another's unhappiness) and shared happiness (i.e., pleasure at another's happiness) (Singer \& Klimecki, 2014). Even though the early ones (i.e., envy and schadenfreude) might be categorized as "negative" feelings, in the sense that they are socially unacceptable, they also have been argued to serve a purpose of human cooperation maintenance and social harmony for long-term individual benefits, ultimately linked to the welfare of social groups (Santamaria-Garcia et al., 2017).

\section{The neural substrates and online electrical responses linked to social feelings of empathy, compassion, envy and schadenfreude}

The study of the neural underpinnings of human sociality, cooperation, and morality has blossomed in recent years (Ibanez et al., 2012). Neuroimaging studies have moved from the study of basic individual emotions (fear, anger, disgust, joy) to the study of rather complex social emotions.

Functional magnetic resonance imaging (fMRI) studies of empathy posit that there is a core network of brain regions underlying empathy across stimuli and tasks (i.e., the observation or evaluation of pain or happiness feelings in others), which includes the cingulate cortex, supplementary motor area, and bilateral anterior insula (see meta-analysis by Fan, Duncan, de Greck, \& Northoff, 2011). Event-related potential (ERP) studies have reported that observing pain inflected in others elicits early responses (i.e., N1, N2 components), presumably tied to an affective sharing response, whereas later components (P3 and LPP) are linked to cognitive evaluation and reappraisal processes. However, a recent meta-analysis by Coll (2018) indicates that only the increasing amplitudes of late components (P3 and LPP) is reliable across studies. Within the framework of economic games, where two people interact to share monetary rewards, the amplitude of the Feedback-Related Negativity (FRN, a 250-300 frontocentral response to the monetary outcome delivery) reflects the distinction between favorable and unfavorable outcomes (i.e., gains and losses) (Gehring \& Willoughby, 2002). The brain component is predictive of the subjective feeling of pleasantness/unpleasantness following monetary outcomes
(Rigoni, Polezzi, Rumiati, Guarino, \& Sartori, 2010). Interestingly, FRN is not only modulated for one's own but for the gains and losses of others in cooperative and antagonistic situations (Itagaki \& Katayama, 2008). In addition, we seem to monitor more the performance of our opponents than the one of other in-group partners in the context of these gambling games (Yu et al., 2019). The FRN also is enhanced when participants receive unfair relative to fair economic offers from others in the cooperative/competitive ultimatum game (Peterburs et al., 2017).

In contrast to empathy, sympathy is sometimes considered a different concept in the sense that understanding but not necessarily sharing another's feelings is involved. The difference according to Lamm, Rutgen, and Wagner (2019) is that sympathy or compassion involves more than a "feeling as" the other but a "feeling for" the other, including concern about him or her. Thus, according to Lamm et al., brain networks engaged during empathy and compassion overlap only scarcely; compassion also engages brain areas linked to the reward system (e.g., studies perform on compassion meditationtrained people). Regarding its timing, empathetic concern has been tied to late rather than early ERP components (Decety, Lewis, \& Cowell, 2015).

Research comparing the neural correlates of absolute and relative losses and gains also offer evidence for the involvement of the prefronto-striatal networks underlying envy and schadenfreude feelings. Displeasure in the fortune of others (envy) recruits increased dorsal anterior cingulate cortex, representing cognitive dissonance, and decreased rewardrelated striatal regions (see review by Jankowski \& Takahashi, 2014). In the ERP field, a preference to attend to envy-related stimuli has been stablished (Zhong, Liu, Zhang, Luo, \& Chen, 2013). Schadenfreude (i.e., joy at others losses or misfortunes) has been associated with activity in the ventral striatum, an area related to reward processing (Jankowski \& Takahashi, 2014; McClure, York, \& Montague, 2004; Takahashi et al., 2009). Nucleus accumbens activity (a part of the striatum) indeed covaries with the intensity of the schadenfreude experience (Paulus et al., 2018). Schadenfreude appears to be implemented very early in human evolution. For example, a cross-species behavioral study shows that both 6-year-olds and chimpanzees are motivated to even incur in personal costs to observe how an antisocial agent, whom they have previously interacted with, gets punished (Mendes, Steinbeis, Bueno-Guerra, Call, \& Singer, 2018). Besides, 5-year-old children act positively toward those who are prosocial and/or negatively toward those who are antisocial to third parties ( $\mathrm{Li} \&$ Tomasello, 2018). Therefore, brain mechanisms have most likely evolved to provide support for the enactment of this kind of social feelings.

Most daily social interactions, however, rather than in the form of monetary exchange occur in the form of information sharing. We often hear information about people: how they 
are, what they do, and what happens to them. How the brain processes incoming verbal information has largely been explored with ERPs (see chapter by Urbach \& Kutas, 2018). When reading sentences for comprehension, smaller N400 amplitudes are elicited by highly expected versus unexpected sentence endings (Kutas \& Federmeier, 2011). Whether this effect reveals that comprehenders predictively commit to specific lexico-semantic predictions is still a debated and unresolved question in the field, with some studies showing evidence in favor (DeLong, Urbach, \& Kutas, 2005; Wicha, Moreno, \& Kutas, 2004) and others against (Nieuwland et al., 2018). Leon-Cabrera et al. (Leon-Cabrera, Flores, Rodriguez-Fornells, \& Moris, 2019; Leon-Cabrera, Rodriguez-Fornells, \& Moris, 2017) provide evidence of context-dependent differences in the state of the system before word reception, while evidence for specific candidate preactivation remains unclear. Beyond the issue of determining what is the precise level of prediction (e.g., phonological, lexical, semantic), an interesting issue is whether social feelings, such as feelings of (un)fairness/deservedness might potentially permeate language comprehension and its temporal dynamics as well. Indeed, general event and world knowledge influence linguistic predictions (Hagoort, Hald, Bastiaansen, \& Petersson, 2004; Metusalem et al., 2012). For example, the identity (Van Berkum, van den Brink, Tesink, Kos, \& Hagoort, 2008) or the reliability of the speaker (Brothers, Dave, Hoversten, Traxler, \& Swaab, 2019) are able to modulate N400 effects.

Our study was designed to explore whether a given "social context" can elicit an emotional reaction that accordingly impacts the processing of a subsequent lexical target. More precisely, do social feelings such as empathy or shared happiness, sympathy or compassion, envy or schadenfreude affect the way we process information about others' social behavior and the subsequent consequences? Do these social feelings affect early fast automatic brain responses (indexing capture of attention), middle range indexes of expectancy or (in) consistency with prior context, or late responses linked to reanalysis processes?

\section{Current study}

Our study used a language comprehension task and ERPs to explore the processing dynamics of lexical input designed to potentially elicit a variety of social feelings. Particularly, we explored whether and how electrical brain responses distinguish between fortunate and unfortunate outcomes delivered to agents previously introduced as displaying either a prosocial or an antisocial behavior. Based on their social profile, whether they end up obtaining some kind of reward or punishment is expected to provoke feelings of empathy (reward of prosocials), pity/compassion (punishment of prosocials), anger/annoyance/envy (reward of antisocials), or even schadenfreude (punishment of antisocials), which ultimately might alter ERP responses at various levels and in various ways. Our analyses focused on the following ERP components: 1) an early P2 effect, related to capture of attentional resources within social statements (Leuthold, Kunkel, Mackenzie, \& Filik, 2015; Van Berkum, Holleman, Nieuwland, Otten, \& Murre, 2009); 2) semantic fit and/or anticipation processes (see review by Kutas \& Federmeier, 2011 on N400 effects); and 3) reanalysis/reevaluation processes indexed by Late Positive Potentials, LPPs (Bayer, Sommer, \& Schacht, 2010; Holt, Lynn, \& Kuperberg, 2009; Kissler, Herbert, Winkler, \& Junghofer, 2009; Schindler, Schettino, \& Pourtois, 2018; Van Berkum et al., 2009). We hypothesized that: 1) unfortunate outcomes would elicit a higher capture of attention than fortunate ones (larger P2); 2) both fortunate outcomes to pro-socials and unfortunate ones to antisocials would elicit the smallest N400 amplitudes by virtue of their higher degree of expectancy; and 3) larger LPPs would be elicited by mismatching (unfortune of prosocials and fortune of antisocials) relative to matching scenarios (fortune of prosocials and unfortune of antisocials). LPP effects also are reported for highly arousing relative to neutral words in language processing tasks (Fischler \& Bradley, 2006; Kissler et al., 2009; Schindler et al., 2018). Thus, perhaps, unfortunate outcomes (potentially more arousing) would elicit larger LPPs than fortunate ones, regardless of the matching or mismatching scenarios.

\section{Methods}

\section{Participants}

A minimum sample size of 15 participants was needed for an estimated effect size of $0.25\left(\mathrm{n}^{2}\right)$ in a minimum set of 4 electrodes (Faul, Erdfelder, Lang, \& Buchner, 2007). For good EEG data quality, however, we aimed for a higher sample size of 20 participants. Twenty native Spanish speakers volunteered to participate in the study in exchange for course credits and economical reward. All participants gave written, informed consent. The study was approved by the local ethics committee and was conducted in accordance with the ethical standards laid down in the Declaration of Helsinki. Data from one participant was excluded from further analysis, because he had less than $40 \%$ valid trials after the EEG artifact rejection procedure. Thus, the final sample included 15 females and 4 males (mean age $=22.1$ years, range $=18-27$ years) . All except for one participant reported being right handed. The average handedness score (Oldfield, 1971) was +70.3 (range, +100 to -50 ). All participants reported normal or corrected-to-normal vision and none had a history of neurological or psychiatric disorders. 


\section{Materials and design}

An initial set of 320 scenarios was created. The topics of the scenarios included the compliance with or the violation of basic social norms (e.g., honesty; courtesy; respect to elderly people, neighbors or coworkers; bullying; cut in lines; vandalism). They were elaborated with the goal that most people would tend to categorize the behavior of the main character as prosocial (altruistic, considerate, cooperative, otherregarding; 160 scenarios) or as antisocial (self-seeking, inconsiderate, uncooperative, selfish; 160 scenarios). Thus, "Carlos is a nice and sensitive boy. He is in love with a girl in his class named Lucia. He is very attentive to her and he reminds her everyday how beautiful she is, " is an example of a prosocial scenario, whereas "Carlos is a selfish and rude boy. He is always bullying Lucia. He is very mean to her and he tells her everyday how ugly she is, " is the pertinent antisocial scenario.

To measure the extent to which our scenarios were categorized as depicting social/antisocial protagonists, the list of scenarios was divided into 5 lists (64 scenarios per list) and 100 subjects ( 20 per list) were asked to evaluate the scenarios in an online web survey. A 5-point rating scale was used, where participants chose 1 when they thought that the protagonist of the scenario was antisocial and 5 when they thought it was prosocial. Prosocial scenarios were rated above 2.5 (mean value $=4.31$, standard deviation $=0.42$ ), and antisocial scenarios were rated below this value (mean value $=1.52$, standard deviation $=0.38$ ). The difference between these two groups of scenarios on prosociality rating was statistically significant $(\mathrm{t}=61.49, p<0.001)$.

We then added to each scenario a short ending sentence in which the protagonist was later on involved in a situation with a fortunate or misfortunate outcome. As a result, four experimental conditions were determined: prosocial protagonists with a fortunate outcome (prosocial-fortunate; tapping into empathy feelings), prosocial protagonists with a misfortunate outcome (prosocial-unfortunate; tapping into pity/compassion feelings), antisocial protagonists with a fortunate outcome (antisocial-fortunate; tapping into anger/annoyance) and antisocial protagonists with a misfortunate outcome (antisocial-unfortunate; tapping into schadenfreude feelings of joy for deservedness/fairness/justice). Sentences were common across conditions except for the last word of the sentence, which determined whether it was a fortunate or a misfortunate outcome. For the prosocial scenario mentioned above (Carlos is a nice and sensitive boy. He is in love with a girl in his class named Lucia. He is very attentive to her and he reminds her everyday how beautiful she is.), a suitable outcome was: "One day, Carlos asks her on a date and she says yes (prosocial fortunate outcome) / no (prosocial unfortunate outcome)." In the pertinent antisocial scenario: "Carlos is a selfish and rude boy. He is always bullying Lucia. He is very mean to her and tells her everyday how ugly she is. One day, Carlos asks her on a date and she says ... , " the "yes" sentence ending represents the antisocial-fortunate condition and the "no" ending would be the antisocial-unfortunate one (see Table 1 for a list of four examples and Appendix for a larger list of examples). $t$ tests were conducted on the fortunate and unfortunate target word endings for the variables "number of letters" and "word frequency of use" (Sebastián-Gallés, Martí, Carreiras, \& Cuetos, 2000). Their mean values did not differ [number of letters; mean fortunate $=7.7$; standard deviation $[\mathrm{SD}]=1.4$; mean unfortunate $=7.7 ; \mathrm{SD}=2.1 ; t=0.025 ; p=0.98$ ) and word frequency of use; mean fortunate $=47.8 ; \mathrm{SD}=9.8 ;$ mean unfortunate $=36.0 ; \mathrm{SD}=4.8 ; t=0.466 ; p=0.64$ ).

Before the ERP recording sessions took place, we conducted a series of norming studies to validate our materials. First, the probability of using a specific word to finish the sentence was computed. In addition, all sentence completions offered by respondents to this norming study were collapsed in terms of whether they were "fortunate" or "unfortunate" endings, beyond what specific word that used to finish the sentence. Finally, the word that was finally selected for the ERP session to complete each sentence was submitted to a norming study on the feelings that it evoked after reading the whole scenario.

In the cloze probability norming study, the last word of each sentence was omitted on purpose for participants to fill it in. Thirty respondents per list were asked to provide the word that first came to their mind after reading the paragraphs and their accompanying sentences. This procedure allowed us to obtain the cloze probability value for the word ending that was later used in the ERP session, as well as an estimate of whether participants tended to finish the sentence with either a fortunate or misfortunate outcome, beyond what particular word was used to finish the sentence.

In addition, we tested the materials (this time including the last word of the ending sentence) on what sort of feelings they elicited. An online survey was conducted on 134 participants (108 females, mean age 19.2 years old [y/o]). Each of 8 lists (including 80 items per list) were created with equal number of items per experimental condition, with no repetitions within a list, and were randomly assigned to participants (15-19 participants per list). Each scenario was presented to be read by participants with the instruction that its out-coming sentence final word needed to be rated in a 1- to 7-point Likert scale on the type of emotion and intensity it elicited to them: where 1 indicated none or very little intensity and 7 indicated the highest intensity. We provided participants with a list of three possible sets of emotions (pleasure/happiness, pity/compassion, and anger/annoyance) together with an open field to indicate (optionally) any other feelings they might need to report. The questionnaire mean time of completion was 30 minutes.

To avoid repetition of scenarios within one participant, for the EEG recording session, the materials also were distributed 
Table 1. Examples of scenarios and outcomes delivered throughout the experiment

Outcome

Fortunate

Unfortunate

Scenario Prosocial Julio is an excellent employee. He does his best at work and his co-workers appreciate him very much.

Antisocial Julio is an awful employee. He gets in late, gets away of his duties, and shouts to his co-workers.

Scenario Prosocial Paula is a nurse at a Hospital. She treats his patients with love and care and sometimes she keeps on working afterhours, especially when there is not enough staff.

Antisocial Paula is a nurse at a Hospital. She treats his patients with disrespect and disdain and always runs away from work early, even if there is not enough staff.

Scenario Prosocial Héctor is a high-school student. There's a lonely boy in his class who's always being bullied. Héctor always steps up to defend him and does his best to avoid his struggling.

Antisocial Héctor is a high-school student. There's a lonely boy in his class who's always being bullied. Héctor thinks that the jokes they play upon him are fun and joins his classmates, insulting him as everybody else does, even though the classmate has never done him any wrong.

Scenario Prosocial Luis stands on trial for a car accident. Actually, he is innocent. He just stopped his car to help the victim.

Antisocial Luis stands on trial for a car accident. Actually, he is guilty, but he argues that he wasn't there, that he was on a trip.
His boss notifies him that His boss notifies him he has been promoted. that he has been fired.

His boss notifies him that His boss notifies him he has been promoted. that he has been fired.

Paula receives an award. Paula receives a sanction.

Paula receives an award. Paula receives a sanction.

One day Héctor is awarded.

One day Héctor is expelled.

One day Héctor is awarded.

One day Héctor is expelled.

When the verdict is When the verdict is delivered he is declared delivered he is absolved. declared guilty.

When the verdict is When the verdict is delivered he is declared delivered he is absolved. declared guilty. in four experimental lists of 160 scenarios each: 40 prosocial scenarios with a fortunate outcome, 40 prosocial scenarios with unfortunate outcome, 40 antisocial scenarios with fortunate outcome, and 40 antisocial scenarios with unfortunate outcome. Thus, each version of a scenario by outcome combination was assigned to one of the four experimental lists. Participants were then randomly assigned to one experimental list, and the presentation order of every paired scenario-ending set was randomized within the list on a trial-by-trial basis. The four-lists procedure guaranteed that each participant never saw both the prosocial and corresponding antisocial scenario within his/her assigned list.

\section{Experimental procedure}

After signing informed consent, participants were fitted with encephalogram (EEG) electrodes while they completed handedness, vision, and health questionnaires. They were seated approximately $100 \mathrm{~cm}$ in front of a 19" computer monitor. The session began with a short set of practice stimuli to acclimate the participants to the silently reading task.

The scenario without its outcome was presented in the screen for unlimited duration. Participants were instructed to read the paragraphs for comprehension and press the space key to initiate the final sentence. The ending sentence appeared word by word in the center of the screen in order to avoid eye movements and obtain precise time-lock to the final word of the sentence. Every word of the sentence was shown in a black 30-point lower-case Arial font on a grey background. Each word was presented for $300 \mathrm{~ms}$, except for the last word, with a duration of $500 \mathrm{~ms}$ to avoid overlapping of the offset of the word on screen with ongoing EEG activity. Inter-words interval lasted $300 \mathrm{~ms}$. In 48 of 160 trials, participants encountered a yes or no question right after the final sentence. This question was related to the previous scenario (e.g., "Is Carlos in love with Lucia?"). Half of the right responses were affirmative, and the other half were negative. Response buttons were "M" and " $Z$," and they were counterbalanced across participants. This procedure was done to ensure participants' attention to the reading task. The averaged hit percentage was $84.4 \%$ (range: $77.08-91.16 \%$ ). No participant data were excluded based on a poor performance on this test. The task was programmed using the Matlab Environment (The MathWorks, Natick, MA) (see Figure 1, for an illustration of the experimental sequence of events).

\section{EEG recording and analysis}

EEG data were recorded from 64 tin electrodes distributed according to the international 10-20 system (American Electroencephalographic Society guidelines for standard electrode position nomenclature, 1991). Electrodes were mounted in an electrode cap (Electro-Cap International). Their impedances were kept below $5 \mathrm{k} \Omega$, referenced online to the left 


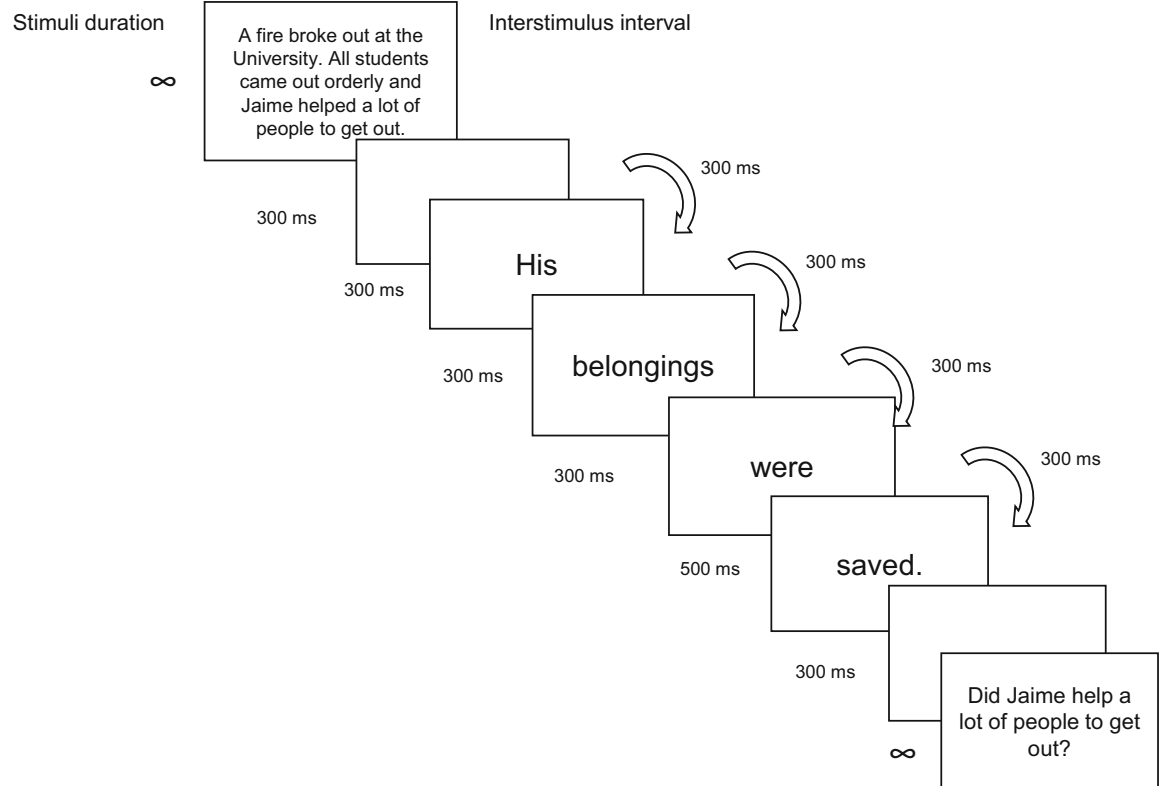

$30 \%$ of trials

Fig. 1. Example of the sequence of events up on the screen during the experiment and the participants' task. This example corresponds to the prosocial-fortunate condition. Trials were divided into 4 blocks, with a

mastoid and amplified with a Brain Amps amplifier at a sampling rate of $1,000 \mathrm{~Hz}$. The signal was filtered through a $0.1-$ $100 \mathrm{~Hz}$ online band-pass filter. The electrooculographic activity was also recorded using vertical and horizontal bipolar electrodes placed at supra-infraorbital level of the left eye and the outer canthus of both eyes, respectively. Data were processed using BrainVision Analyzer software (Brain Products, Munich). The averaged voltage of the right and left mastoids was used as the offline reference. For artifact rejection purposes, the following thresholds were set: maximal allowed voltage step, $50 \mu \mathrm{V}$; minimal and maximal allowed amplitude, $\pm 100 \mu \mathrm{V}$; lowest allowed activity (max-min), $5 \mu \mathrm{V}$ for a 1,500-ms interval length. Once any of these thresholds was met in the continuous EEG file, data recorded at that point were marked and discarded, together with data recorded during $200 \mathrm{~ms}$ before and after detection. This method was performed to avoid including any residual artifacts in subsequent computations of ERP averages. EEG raw data from all subjects were scanned and marked using the same criteria. The number of remaining trials for one participant after artifacts removal was below 15 (37.5\% of trials) and was discarded for this reason. The number of trials was above this threshold for the rest of participants. The percentage of remaining trials per condition was $80.75 \%$ for prosocial scenarios with a fortunate outcome, $77.63 \%$ for prosocial scenarios with an unfortunate outcome, $80.25 \%$ for antisocial scenarios with an unfortunate outcome, and $78.5 \%$ for antisocial scenarios with a fortunate outcome. No differences between the number of remaining trials per condition were found $[\mathrm{F}(3,72)=0.146, p=0.932]$.

A Butterworth zero phase filter was applied to the EEG data (low cutoff at $0.1 \mathrm{~Hz}$, time constant $=1.6 \mathrm{~s}, 24 \mathrm{db} / \mathrm{oct}$; break between them. Break's duration was unlimited; participants decided when to start the next block. The whole session lasted about an hour

high cutoff at $20 \mathrm{~Hz}, 24 \mathrm{db} / \mathrm{oct}$ ). The continuous EEG was segmented into 1,000-ms epochs starting $100 \mathrm{~ms}$ before the onset of the target word. Artifact-free average waveforms were then computed for each condition separately, after subtraction of the prestimulus baseline. The following scalp Regions of Interest (ROIs) and set of electrodes were defined: Right Anterior (RA: electrodes F4, F6, F8, FC4, FC6); Left Anterior (LA: F3, F5, F7, FC3, FC5); Midline Anterior (MA: F1, Fz, F2, FC1, FCz, FC2); Right Central (RC: C4, C6, CP4, CP6, T8; TP8); Left Central (LC: C3, C5, CP3, CP5, T7, TP7); Midline Central (MC: C1, Cz, C2, CP1, CPz, CP2); Right Posterior (RP: P6, P8, PO6, PO8, O2); Left Posterior (LP: P5, P7, PO5, PO7, O1); and Midline Posterior (MP: P1, $\mathrm{Pz}, \mathrm{P} 2, \mathrm{POz}, \mathrm{Oz})$. We conducted repeated-measures analysis of variance (ANOVAs) involving the factors: type of scenario (prosocial/antisocial), type of outcome (fortunate/unfortunate for P2 and LPP effects and congruent/incongruent for N400 effect), and ROI (9 levels). A Huynh-Feldt correction was applied when evaluating effects with more than one degree of freedom. A Bonferroni correction was used for multiple comparisons.

\section{Results}

\section{Cloze probability test}

Cloze probability of expected outcomes was low [Prosocialfortunate: $17 \%(\mathrm{SD}=24.3)$ and Antisocial-unfortunate: $16.8 \%(\mathrm{SD}=24.8)]$. As expected, it was even lower for unexpected outcomes [Prosocial-unfortunate: $6.8 \%(\mathrm{SD}=14.8)$ 
vs. Antisocial-fortunate: $5.2 \%$ ( $\mathrm{SD}=12.7)]$. No statistical differences emerged within each level of expectancy (i.e., within expected or unexpected outcomes; $p \mathrm{~s}=0.95$ and 0.27 , respectively). All other comparisons across levels of expectancy were significant (all $p \mathrm{~s}<0.001$ ).

In addition, tendencies to expect either fortunate or unfortunate outcomes overall (beyond the specific lexical item that was used by participants to complete the sentence) was also assessed. Those tendencies were computed by assigning a value of positiveness or negativeness to the answers provided by the respondents to the questionnaire. Thus, for example, for the scenario "Raúl is a very tranquil person. When we are at the subway platform and the train arrives to the station, he lets passengers out with calm before he goes in. When we are inside the train Raúl receives a/an ..." responses, such as "seat," "applause," and "candy," were all considered positive fortunate outcomes, whereas "shove," "stamp," "nudge," or "reprimand" were all considered negative unfortunate outcomes. In the mirroring scenario: "Raúl is always going around rushing and overrunning. When we are at the subway station as soon as the train arrives and the doors open, he makes his way in by pushing others not letting them out. When we are inside the train Raúl receives alan ..." same criteria was used to classify emitted responses as positive or negative outcomes. According to these measurements, participants tended to expect fortunate rather than unfortunate outcomes ( $74.95 \%$ and $25.05 \% ; \pm 3.71 \%$, respectively) to prosocial protagonists. The opposite was true for antisocial protagonists (higher expectation for unfortunate than fortunate outcomes, $79.18 \%$ and $20.82 \% ; \pm 3.32 \%$, respectively).

Responses to the feelings questionnaire were submitted to a mixed ANOVA, including the between variable experimental condition (4 levels) and the within variable intensity of feelings (4 levels). Post hoc Bonferroni corrected paired $t$ tests revealed that pleasure/happiness was the highest emotion within prosocial fortunate scenarios, whereas pity/ compassion was the highest in the prosocial unfortunate scenarios (followed by anger/annoyance feelings). For the antisocial scenarios, anger/annoyance was highest than pleasure/ happiness or pity/compassion (no significant differences between the latest) for fortunate outcomes. By contrast, antisocial scenarios with unfortunate outcomes elicited to the maximum intensity pleasure/happiness (schadenfreude feelings), followed by anger/annoyance and pity/compassion feelings (all $p$ values $<0.02$; Figure 2).

\section{Event-related potentials}

Visually, a negative going deflection at around $400 \mathrm{~ms}$ was observed, where the antisocial unfortunate condition showed a smaller negative amplitude than the rest of conditions at parieto-occipital electrodes (Figure 3A, P4 electrode). This pattern was, in contrast, not observed at frontal electrodes
(Figure 3A, FC4 electrode), suggesting that this effect had the scalp distribution of an N400 effect (Figure 3B; N400).

\section{P2}

Based on modulations observed in the $200-250$ ms latency range by Van Berkum et al. (2009) and Leuthold et al. (2015) for P2 effects, we submitted our results to a repeatedmeasures analysis (ANOVA), involving the factors type of scenario (prosocial, antisocial), type of outcome (fortunate, unfortunate), and ROI (9 levels). The analysis revealed a main effect of ROI $\left[\mathrm{F}(8,144)=5.58 ; p=0.004 ; \mathrm{y}_{\mathrm{p}}^{2}=0.237\right]$, with mean amplitude oscillations from $0.62 \mu \mathrm{V}$ in the LC ROI to $3.42 \mu \mathrm{V}$ in the RP ROI. Analysis at each ROI revealed that type of scenario exerted an effect in RA and RC ROIs [F(1, 18) $=4.84 ; p=0.041 ; \mathrm{\eta}_{\mathrm{p}}^{2}=0.212$ and $\mathrm{F}(1,18)=6.37 ; p=$ $0.021 ; \eta^{2}=0.261$, respectively].

In both right scalp regions, brainwaves elicited by outcomes to prosocial characters were more positive (i.e., larger P2) compared with those elicited by outcomes to antisocial ones.

\section{N400}

We measured mean amplitude values in the $375-500 \mathrm{~ms}$ interval. The time-window was selected to avoid picking up earlier P2 and later developing LPP oscillations. We submitted these values to a repeated-measures ANOVA, involving the factors type of scenario (prosocial, antisocial), type of outcome (congruent, incongruent), and ROI (9 levels). A main effect of ROI revealed mean amplitude differences across electrode regions $\left[\mathrm{F}(8,144)=3.66 ; p=0.024 ; \mathrm{\eta}_{\mathrm{p}}^{2}=0.169\right]$. Analysis at each region revealed that as expected, congruent outcomes (i.e., prosocial-fortunate and antisocial-unfortunate) elicited less negative mean amplitude responses than incongruent ones (i.e., prosocial-unfortunate and antisocial-fortunate), i.e., type of outcome effect [LP region: $\mathrm{F}(1,18)=4.72 ; p=0.043 ; \mathrm{\eta}^{2} \mathrm{p}=$ $0.208 ; \mathrm{RP}$ region $\left.\mathrm{F}(1,18)=4.78 ; p=0.042 ; \mathrm{y}_{\mathrm{p}}^{2}=0.210\right]$. The type of outcome effect also was marginally significant at RC, LC, and MP regions ( $p$ values between 0.059 and 0.067). In addition, a main effect of type of scenario was also obtained at $\mathrm{LP}$ and RP regions $\left[\mathrm{F}(1,18)=4.84 ; p=0.041 ; \mathrm{n}^{2} \mathrm{p}=0.212\right.$ and $\mathrm{F}(1,18)=7.17 ; p=0.015 ; \mathrm{\eta}_{\mathrm{p}}^{2}=0.285$, respectively], with a reduced N400 for social than antisocial scenarios (also marginal at MP region).

The interaction between type of scenario and type of outcome was significant in the MP region $[\mathrm{F}(1,18)=4.64 ; p=$ $\left.0.045 ; \mathrm{y}_{\mathrm{p}}^{2}=0.205\right]$ and marginally significant in the RP region $\left[\mathrm{F}(1,18)=3.81 ; p=0.067 ; \mathrm{n}_{\mathrm{p}}^{2}=0.175\right]$. In the MP ROI, post-hoc tests revealed significantly less negative voltage values (i.e., reduced N400) for the antisocial unfortunate condition than the rest of conditions [prosocial fortunate $(p=$ $0.016)$; prosocial unfortunate $(p=0.018)$; and antisocial 


\section{Feelings Questionnaire}

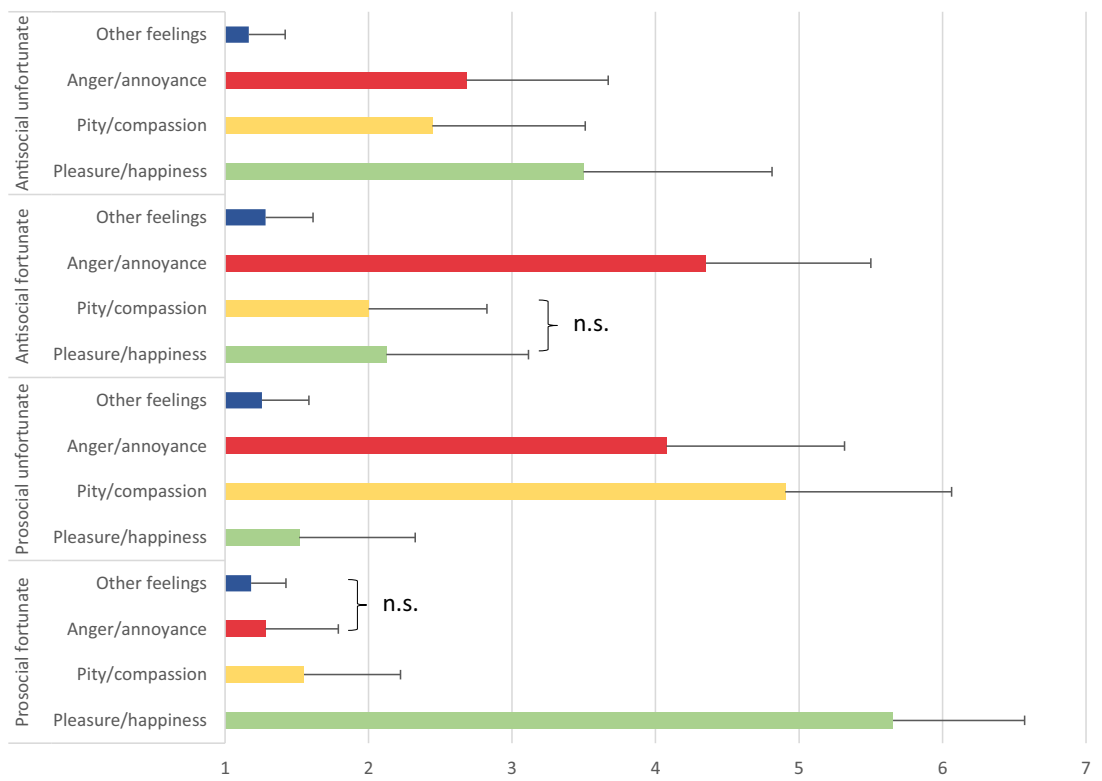

Fig. 2. Feelings Questionnaire. Intensity of emotions (from $1=$ none/ lowest to $7=$ highest) in the $\mathrm{X}$-axis that was reported by participants in the Feelings Questionnaire by experimental condition (in the Y-axis).

fortunate $(p=0.019)]$. Thus, the processing of an unfortunate outcome for an antisocial protagonist was associated with a reduced N400 (i.e., facilitated processing), relative to all other
Within each condition, paired $t$ tests revealed significant mean differences (all $p \mathrm{~s}<0.02$ ), except when specified with n.s. (nonsignificant) brackets.

possible outcomes, which might be linked to feelings of schadenfreude, as we will discuss later. The amplitude of the response to fortunate or unfortunate outcomes to prosocial a

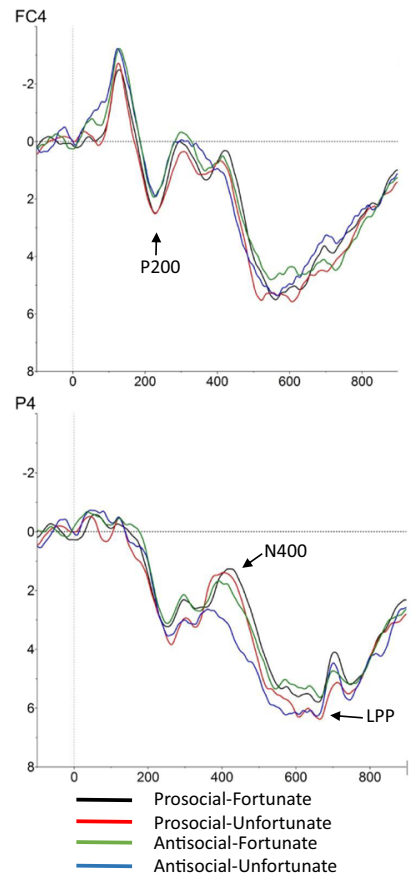

b

P200

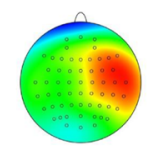

Antisocial - Prosocial

$200 \mathrm{~ms}-250 \mathrm{~ms}$

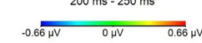

N400

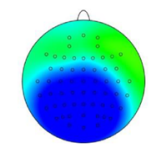

Prosocial Fortunate Antisocial Unfortunate

$375 \mathrm{~ms}-500 \mathrm{~ms}$

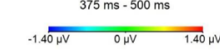

LPP

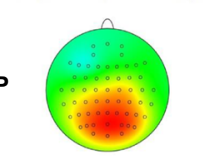

Unfortunate - Fortunate

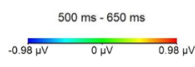

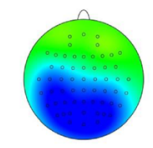

Prosocial Unfortunate Antisocial Unfortunate

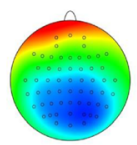

Antisocial Fortunate - Prosocial Fortunate Antisocial Unfortunate Prosocial Unfortunate

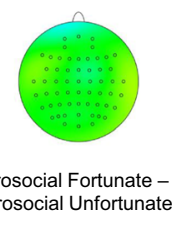

to a prosocial relative to an antisocial protagonist. At the parietal electrode, an N400 was elicited by all conditions, except for the antisocial-unfortunate condition. In the same electrode, $\mathrm{P} 4$, at a later time-window an LPP enhancement is observed for unfortunate relative to fortunate outcomes. B. Voltage maps show the distribution of each of these effects across the scalp (difference brainwaves). 
protagonists was, by contrast, not modulated in this timewindow ( $p=0.652$; Fig. 4, left panel).

\section{LPP}

Finally, in line with reports of LPP modulations we extracted the mean amplitude values in the 500-650 ms time-window, the same latency range used by Van Berkum et al. (2009), and submitted them to repeated-measures analysis. A main effect of ROI emerged $\left[\mathrm{F}(8,144)=9.51 ; p<0.001 ; \mathrm{y}_{\mathrm{p}}^{2}=0.346\right]$, with mean amplitudes oscillating between 3.9 and $5.9 \mu \mathrm{V}$ depending on the ROI. Analysis at each ROI revealed a marginal main effect of type of outcome in the MP region $[\mathrm{F}(1$, $18)=4.34 ; p=0.052 ; \mathrm{j}_{\mathrm{p}}^{2}=0.194$ [with larger LPP responses to unfortunate $(5.8 \mu \mathrm{V})$ than fortunate outcomes $(4.9 \mu \mathrm{V})]$.

\section{Discussion}

The measurement of feelings evoked by our sentence materials revealed that the highest reported emotion to prosocial protagonists was pleasure/happiness when they received fortunate outcomes and pity/compassion when they received unfortunate ones (followed by anger/annoyance in the later). With regard to antisocial protagonists, fortunate outcomes elicited higher anger/annoyance feelings, whereas unfortunate ones elicited mostly pleasure/happiness (schadenfreude) feelings, followed by anger/annoyance and pity/compassion as well.

Not surprisingly, according to the cloze probability task, prosocial characters were expected to receive fortunate

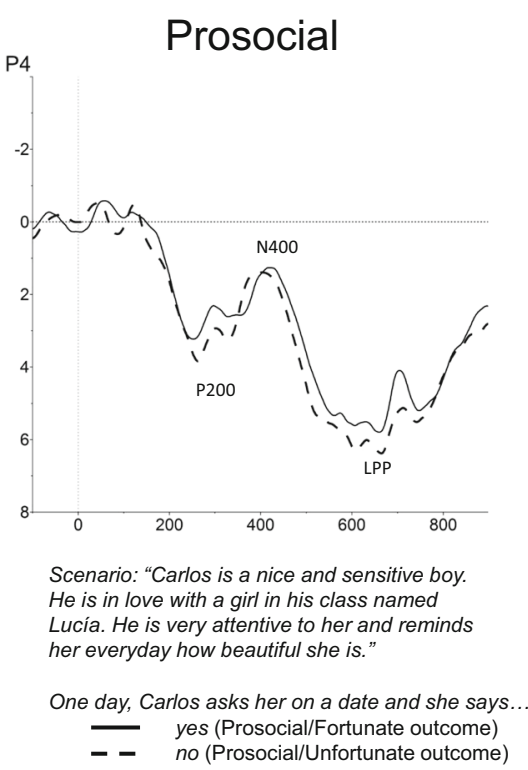

Fig. 4. Brainwave responses are shown separately for prosocial (left) and antisocial (right) protagonists at the P4 electrode. Negative voltage is plotted up in the Y-axis. Time 0 , in the $\mathrm{X}$-axis, indicates the onset of the critical final word of the sentence. The N400 response was similar for outcomes to a higher extent than unfortunate ones, and the opposite was true for antisocial characters. Despite no distinction could be made between the high expectation of a fortunate outcome for a prosocial character and the high expectation of an unfortunate one for an antisocial one ( $80 \%)$. ERP measurements offered a more complete and detailed picture of the temporal unfolding of target word processing. During the online reading task, we randomly provided fortunate/ unfortunate outcomes (low cloze probability ones) to the scenario protagonists. In line with the wide literature on N400 effects, these low cloze probability words elicited an N400 enhancement (see Kutas \& Federmeier, 2011, for a review). Remarkably, N400 amplitude was not only modulated by lexical expectancy (i.e., cloze probability alone) but also depending on the particular scenario and the type of outcome. Additionally and in consonance with the still scarce ERP literature on "social" factors influencing language processing, modulations also were observed in P2 and LPP ERP components (Leuthold et al., 2015; Schacht \& Vrticka, 2018; Van Berkum et al., 2009).

At a first stage ( $\sim 200 \mathrm{~ms})$, outcomes delivered to prosocial and antisocial characters diverged. Thus, in line with previous ERP studies on discourse processing of moral scenarios (Leuthold et al., 2015; Van Berkum et al., 2019), a P2 modulation was observed. Specifically, a larger fronto-central right lateralized P2 was elicited by outcomes to prosocial versus antisocial protagonists, regardless of whether the outcome was fortunate or unfortunate. This result goes against our initial prediction that unfortunate outcomes would capture more attention than fortunate ones. Our fronto-central right P2 effect was instead modulated in relation to the "kindness" or

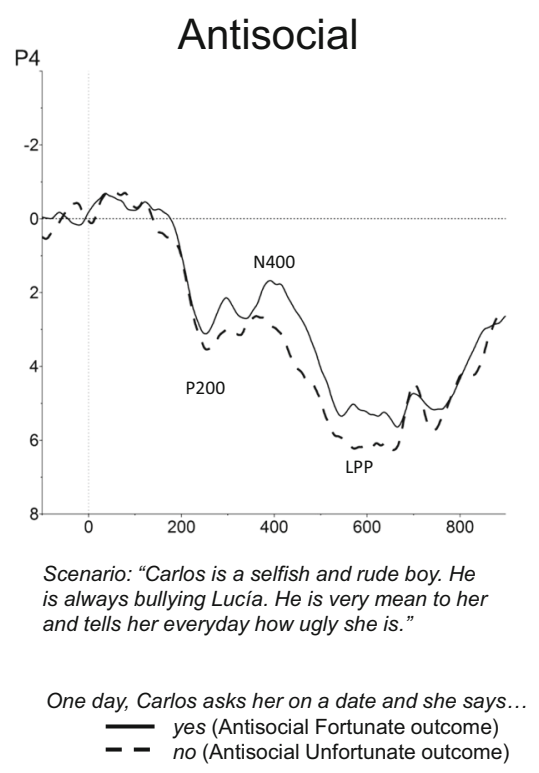

prosocial protagonists, regardless of whether the outcome was fortunate or unfortunate (left). The N400 response was reduced for antisocial protagonists with an unfortunate versus a fortunate outcome (right). 
"unkindness" of the protagonist (prosocial or antisocial). This result most likely indicates that the reading of what ends up happening to a prosocial character (before it gains a more profound lexical access, that is, prior to the N400 component) had a higher attentional capture in participants, relative to what ends up happening to an antisocial one. The functional significance of this effect could be that, as social beings, we care more or give initial priority to what happens to prosocial than antisocial characters. In this vein, an enhancement of early ERP responses also has been found for social compared to non-social contents using complex visual scenes (Schacht \& Vrticka, 2018).

After this initial capture of attentional resources, later latency ERP modulations (at N400 and LPP components) offer a different picture, particularly with consequences for the processing of unfortunate outcomes to anti-social protagonists (N400) and for the processing of unfortunate outcomes as a whole, that is, regardless of the character's social profile (LPP).

The ERP component that is most sensitive to contextual cloze probability (the $\mathrm{N} 400$ ) revealed that participants showed the most reduced N400 amplitude in response to unfortunate outcomes for antisocial protagonists, indicating their facilitated processing as we hypothesized. Thus, interestingly, and in line with the functional significance of N400 ERP amplitude reductions (Kutas \& Federmeier, 2011; Leon-Cabrera et al., 2017), our data indicate that some sort of "punishment" was easier to process and/or more anticipated in those scenarios. It is even more remarkable given that cloze probability for all specific target words (including this one) was always low $(\sim 11 \%)$, and thus all outcomes were bound to elicit an equally large N400 response. According to the results of the feelings questionnaire, the schadenfreude condition elicited the highest intensity in the pleasure/happiness domain. Thus, we speculate that the N400 reduction might tap into "schadenfreude" feelings in the sense that readers were perhaps more "eager" (i.e., anticipated to a higher degree and/or more easily integrated) that an antisocial character had a pertinent future bad outcome. A misfortune to them did not come as a surprise. Somehow, "he had it coming," so to speak. However, against our hypothesis the results with the fortunate outcomes to prosocial protagonists, also scoring high in the pleasure/ happiness feelings, which did not show this reduction in the amplitude of the N400 response. According to our paper-andpencil tasks, the expectation of an any kind of unfortunate outcome for an antisocial character $(79.18 \%)$ was not significantly higher than the expectation of any fortunate outcome for a prosocial one $(74.95 \%)(\mathrm{t}=-1.55, p=0.124)$. Thus, both might have elicited similarly small N400 responses, which was not what we found. We speculate that in the context of unpredictably filled or unfulfilled expectations, participants somehow held more automatic/stronger expectations for unfortunate outcomes to antisocials than fortunate outcomes to prosocials, being responsible for the N400 reduction of the first and not the second. Paper-and-pencil offline tasks are perhaps not able to capture the contrast relative to online brain measures. In an ERP study of optimistic versus pessimistic predictions, a similar phenomenon occurred. Despite an equally high cloze probability, participants seem to refrain more from making strong optimistic versus pessimistic predictions online (Moreno \& Vazquez, 2011). Future studies are needed to establish more clearly whether N400 amplitudes reflect more than just the mathematical probability of lexical inputs, including perhaps the participants' willingness/ eagerness to commit to strong predictions online when the incoming input is not only uncertain but additionally involves emotional consequences.

Although the view of N400 as a sign of lexical anticipatory processes is still controversial (Kuperberg \& Jaeger, 2016; Nieuwland et al., 2018), our N400 results (whether indexing semantic integration facilitation and/or anticipatory processes) are in line with findings in the social behavior literature that early in onto- and phylogenetic development, humans are motivated to see punishment enacted on antisocial agents (Mendes et al., 2018). An alternative explanation may be envisaged. Unfortunate and fortunate outcomes might have differed in their degree of internal semantic closeness or similarity. That is, perhaps the negative outcomes, "shove," "stamp," "nudge," and "reprimand," shared more semantic features in common than "seat," "applause," and "candy" (positive outcomes) did, and their higher degree of higher semantic similarity might have been responsible for the N400 reduction. However, we are reluctant to accept at present such alternative explanation. A higher degree of semantic closeness between negative versus positive lexical entries is not a well-established finding. In fact reports of the opposite (i.e., positive stimuli being generally more similar to one another than negative stimuli are) can be found in the literature (Koch, Alves, Kruger, \& Unkelbach, 2016).

In addition, in the post-N400 time-window, a trend was found for unfortunate outcomes increasing the LPP response, for both prosocial and antisocial characters (MP region). Previous research obtained larger LPPs to conditions that clashed with moral norms of the reader (i.e., moral values inconsistent words) (Van Berkum et al., 2009). In our study, the late response was increased in readers to unfortunate sentence endings regardless of the sociality profile of the protagonist. This result may be in line with the extended finding that highly arousing stimuli tend to generate larger LPP responses than neutral ones in language processing tasks (Fischler \& Bradley, 2006; Kissler et al., 2009; Schindler et al., 2018). Our target words were custom made to fit the specific scenarios and thus were not valuated on valence and arousal ratings. Nonetheless, by definition, unfortunate word outcomes most 
likely had a negative valence value, whereas fortunate ones had a positive valence; their arousal levels are less transparent. Our results replicate prior ones in which the processing of emotional words in emotionally neutral contexts increase late ERP positivities, particularly for negative and highly arousing words in context (Bayer et al., 2010) and negative versus positive words (Holt et al., 2009). Our study is thus inconclusive as to whether LPP modulations are due to valence and/or arousal factors. A higher order explanation is that both negative and/or highly arousing stimuli in context required an extended reanalysis or reevaluation process. In fact, according to recent views, the LPP can be used as a measure of emotion regulation (Hajcak, MacNamara, \& Olvet, 2010), in the sense that upregulation of emotion increases its amplitude while downregulation decreases it (Langeslag \& Sanchez, 2018; Langeslag \& van Strien, 2018). Future studies will help to elucidate these issues.

\section{Conclusions}

The present data shed new light onto the online unfolding of social factors during a reading for comprehension task. We propose that the P200, N400, and LPP modulation patterns obtained in our study relate, respectively, to: 1) a higher capture of attention to whatever happens to prosocial versus antisocial individuals; 2) a good semantic fit for unfortunate outcomes delivered to antisocial ones (processed as a good "match," perhaps tapping into schadenfreude feelings); and 3) an emotional evaluation or reappraisal for unfortunate endings versus fortunate endings, regardless of whether the character was prosocial or antisocial. Accordingly, online psychophysiological responses reveal that the initial response (a frontal P2 enhancement) indicates that outcomes to prosocial characters became more salient and attracted attention. Later on, learning that an antisocial character was confronted with some sort of "punishment" does not come as a surprise (an N400 to a low cloze probability word ending is not elicited). Our data suggest that feelings of schadenfreude (i.e., pleasure, joy, or self-satisfaction) to an antisocial character's misfortune lie behind this electrophysiological diminished amplitude N400 response by virtue of making unfortunate outcomes rather expected. Finally, all negatively valenced unfortunate outcomes seem to incur in reevaluation and reappraisal processes (larger LPP). Together, these effects indicate not only that social feelings immediately and inevitably permeate language comprehension, but also that sociability experience develops across different temporal stages with remarkable specificity.
Acknowledgments The authors thank all study participants for their cooperation. This research was supported by Ministerio de Economia y Competitividad (MINECO) [grant numbers: PSI2014-60682-R and PGC2018-098876-B-100 to EMM, PSI2017-82357-P to MML, and PSI2014-51890-C2-1-P to FC]. PRG was supported by a Complutense University pre-doctoral scholarship.

\section{Compliance with ethical standards}

Declarations of interest None.

\section{Appendix}

All scenarios (160) and their corresponding continuing sentences were presented in Spanish. This appendix provides 18 examples translated into English.

Scenario 1:

- Social: Carlos is a nice and sensitive boy. He is in love with a girl in his class named Lucía. He is very attentive to her and reminds her everyday how beautiful she is.

- Antisocial: Carlos is a selfish and rude boy. He is always bullying Lucía. He is very mean to her and tells her everyday how ugly she is.

Common final sentence with two plausible endings: One day, Carlos asks her on a date and she says ... yes/no. Scenario 2:

- Social: Julio is an excellent employee. He does his best at work and his coworkers appreciate him very much.

- Antisocial: Julio is an awful employee. He gets in late, gets away of his duties, and shouts to his coworkers.

Common final sentence with two plausible endings: His boss notifies him that he has been ... promoted/fired. Scenario 3:

- Social: Paula is a nurse at a hospital. She treats her patients with love and care and sometimes she keeps on working after hours, especially when there is not enough staff.

- Antisocial: Paula is a nurse at a hospital. She treats her patients with disrespect and disdain and always runs away from work early, even if there is not enough staff.

Common final sentence with two plausible endings:

Paula receives a(n) ... award/sanction.

Scenario 4:

- Social: Alberto is lovely to his mother. He always gives her a ride to medical appointments and joins her at the doctor's office to make her feel calm. He always looks after her and worries about all her needs. 
- Antisocial: Alberto is very insensitive to his mother. If she tells him that she has a doctor's appointment, he forgets about it and never asks how things went at the doctor's office. He does not care about her at all.

Common final sentence with two plausible endings:

Alberto has a lot of ... friends/enemies.

Scenario 5:

- Social: Roberto has self-control. Even if he gets angry, nobody notices it, because he is very patient. He looks for solutions to any problem and never gets upset.

- Antisocial: Roberto has a bad temper. The other day he got angry for no reason and started to beat some garbage cans. Then, he left them thrown away in the middle of the street.

Common final sentence with two plausible endings:

Roberto is very ... well-respected/unpopular.

Scenario 6:

- Social: Álvaro enjoys his free time offering private lessons to kids having school difficulties. He does it for free, because teaching is his true vocation. Most of his students have improved their performance.

- Antisocial: Álvaro works at a private academy teaching kids with school difficulties. He does not like teaching. During his classes, he goes no further than delivering dictates and ordering assignments, and his students show not a single sign of improvement.

Common final sentence with two plausible endings:

Álvaro receives from his student's parents many ... congratulations/complaints.

Scenario 7:

- Social: Helena has a lot of doubts about next Friday's exam subject matter, but her classmate Beatriz decides to help her and solve them all.

- Antisocial: Helena has many doubts about next Friday's exam subject matter and asks her classmate Beatriz for some help. She says that it is her own problem and does not help her.

Common final sentence with two plausible endings:

When exam scores are released Beatriz finds out that hers is ... satisfactory/unsatisfactory.

Scenario 8:

- Social: Adriana receives a phone call from a friend. She is very concerned, because she made a big mistake and feels very embarrassed about it and asks Adriana to keep her secret. Adriana, without hesitation, keeps the secret to herself and never tells anyone.
- Antisocial: Adriana receives a phone call from a friend. She is very concerned, because she made a big mistake and feels very embarrassed about it and asks Adriana to keep her secret. Immediately, Adriana calls everyone to tell her friend's secret.

Common final sentence with two plausible endings:

The next day Adriana receives a ... check/fine.

Scenario 9:

- Social: We created a dropbox account in our class to share our class notes. Alejandro always attends classes and shares his notes.

- Antisocial: We created a dropbox account in our class to share our class notes. Alejandro never uploads anything and he is always asking others to upload files for all the classes that he skips.

Common final sentence with two plausible endings:

Alejandro always ... succeeds/flunks.

Scenario 10:

- Social: Carolina is a physician at a private clinic. She is very friendly, and when her visits finish, she provides a clear and explicative invoice to her clients. Antisocial: Carolina is a physician at a private clinic. She isn't very friendly, and when her visits finish, she does not provide an invoice, overcharging her clients without any explanation.

Common final sentence with two plausible endings:

Carolina's clinic has many ... earnings/looses.

Scenario 11:

- Social: Héctor is a high school student. There is a lonely boy in his class who is always being bullied. Héctor always steps up to defend him and does his best to avoid his struggling.

- Antisocial: Héctor is a high school student. There is a lonely boy in his class who is always being bullied. Héctor thinks that the jokes they play on him are fun and joins his classmates, insulting him as everybody else does, even though the classmate has never done him any wrong.

Common final sentence with two plausible endings:

One day Héctor is ... awarded/expelled.

Scenario 12:

- Social: Carlos is a very cautious driver. He never drinks when he has to drive. However, the other day, he had an accident. 
- Antisocial: Carlos is a reckless driver. He always drinks even if he has to drive afterwards. The other day, he had an accident.

Common final sentence with two plausible endings: In the accident he came out ... unharmed/ejected. Scenario 13:

- Social: Sandra is not the kind of girl that pays attention to other people's looks, but she often makes compliments to her girlfriends when she likes what they are wearing. Yesterday, she went to the hairdresser.

- Antisocial: Sandra is always on top of everybody else's looks and criticizes with disdain whatever her girlfriends are wearing. Yesterday, she went to the hairdresser.

Common final sentence with two plausible endings:

Her hairstyle came out ... amazing/awful.

Scenario 14:

- Social: A fire broke out at the university. All of the students came out orderly, and Jaime helped many people to get out.

- Antisocial: A fire broke out at the university. All of the students came out rowdily, and Jaime made his way out by jostling others to get out first.

Common final sentence with two plausible endings:

His belongings were/got ... saved/burned.

Scenario 15:

- Social: Teresa and her schoolmates spend all afternoon working on a school project. Teresa was in charge of writing up the contents of the file and finishing at home what was left to be done.

- Antisocial: Teresa didn't feel like working on the school project. She asked her schoolmates for a file with what they had done and copied it all entirely.

Common final sentence with two plausible endings:

When she was about to close the document, the file was/got ... saved/deleted.

Scenario 16:

- Social: Luis stands on trial for a car accident. Actually, he is innocent. He just stopped his car to help the victim.

- Antisocial: Luis stands on trial for a car accident. Actually, he is guilty, but he argues that he wasn't there but that he was on a trip.

Common final sentence with two plausible endings: When the verdict is delivered he is declared ... absolved/ guilty.
Scenario 17:

- Social: Hugo has applied for a fellowship. He has a short curriculum, but he has been honest, listing only those courses that he has attended to.

- Antisocial: Hugo has applied for a fellowship. Because he has never done anything, he made up courses and seminaries that he never took in a forged curriculum.

Common final sentence with two plausible endings: In the selected applicants list is ... Hugo/someone else. Scenario 18:

- Social: Luis is walking around. An old lady trips over and falls down. Luis offers her some help and accompanies her home to make sure she is all right.

- Antisocial: Luis is walking around. An old lady trips over and falls down. Luis laughs at her and tells her to go and get a walker.

Common final sentence with two plausible endings:

The next day, Luis' mother tells him that his allowance will ... increase/lower.

None of the data or materials for the experiments reported here is available, and none of the experiments was preregistered. Materials and data will be available upon request.

\section{References}

Bayer, M., Sommer, W., \& Schacht, A. (2010). Reading emotional words within sentences: the impact of arousal and valence on event-related potentials. International Journal of Psychophysiology, 78(3), 299307. doi:https://doi.org/10.1016/j.ijpsycho.2010.09.004

Brothers, T., Dave, S., Hoversten, L. J., Traxler, M. J., \& Swaab, T. Y. (2019). Flexible predictions during listening comprehension: Speaker reliability affects anticipatory processes. Neuropsychologia, 135, 107225. doi:https://doi.org/10.1016/j. neuropsychologia.2019.107225

Coll, M. P. (2018). Meta-analysis of ERP investigations of pain empathy underlines methodological issues in ERP research. Social Cognitive and Affective Neuroscience, 13(10), 1003-1017. doi:https://doi.org/ $10.1093 / \mathrm{scan} / \mathrm{nsy} 072$

Decety, J., Lewis, K. L., \& Cowell, J. M. (2015). Specific electrophysiological components disentangle affective sharing and empathic concern in psychopathy. Journal of Neurophysiology, 114(1), 493504. doi:https://doi.org/10.1152/jn.00253.2015

DeLong, K. A., Urbach, T. P., \& Kutas, M. (2005). Probabilistic word pre-activation during language comprehension inferred from electrical brain activity. Nature Neuroscience, 8(8), 1117-1121. doi:https:// doi.org/10.1038/nn1504

Fan, Y., Duncan, N. W., de Greck, M., \& Northoff, G. (2011). Is there a core neural network in empathy? An fMRI based quantitative metaanalysis. Neuroscience and Biobehavioral Reviews, 35(3), 903-911. doi:https://doi.org/10.1016/j.neubiorev.2010.10.009

Faul, F., Erdfelder, E., Lang, A. G., \& Buchner, A. (2007). G*Power 3: a flexible statistical power analysis program for the social, behavioral, 
and biomedical sciences. Behav Res Methods, 39(2), 175-191. Retrieved from http://www.ncbi.nlm.nih.gov/pubmed/17695343

Fehr, E., \& Schurtenberger, I. (2018). Normative foundations of human cooperation. Nature Human Behaviour, 2(7), 458-468. Retrieved from < Go to ISI $>$ ://WOS:000446612000015

Fischler, I., \& Bradley, M. (2006). Event-related potential studies of language and emotion: words, phrases, and task effects. Progress in Brain Research, 156, 185-203. doi:https://doi.org/10.1016/S0079. 6123(06)56009-1

Gehring, W. J., \& Willoughby, A. R. (2002). The medial frontal cortex and the rapid processing of monetary gains and losses. Science, 295(5563), 2279-2282. doi:https://doi.org/10.1126/science. 1066893

Hagoort, P., Hald, L., Bastiaansen, M., \& Petersson, K. M. (2004). Integration of word meaning and world knowledge in language comprehension. Science, 304(5669), 438-441. doi:https://doi.org/ 10.1126/science. 1095455

Hajcak, G., MacNamara, A., \& Olvet, D. M. (2010). Event-related potentials, emotion, and emotion regulation: an integrative review. Developmental Neuropsychology, 35(2), 129-155. doi:https://doi. org/10.1080/87565640903526504

Holt, D. J., Lynn, S. K., \& Kuperberg, G. R. (2009). Neurophysiological correlates of comprehending emotional meaning in context. Journal of Cognitive Neuroscience, 21(11), 2245-2262. doi:https://doi.org/ 10.1162/jocn.2008.21151

Ibanez, A., Melloni, M., Huepe, D., Helgiu, E., Rivera-Rei, A., CanalesJohnson, A., ... Moya, A. (2012). What event-related potentials (ERPs) bring to social neuroscience? Social Neuroscience, 7(6), 632-649. doi:https://doi.org/10.1080/17470919.2012.691078

Itagaki, S., \& Katayama, J. (2008). Self-relevant criteria determine the evaluation of outcomes induced by others. Neuroreport, 19(3), 383387. doi:https://doi.org/10.1097/WNR.0b013e3282f556e8

Jankowski, K. F., \& Takahashi, H. (2014). Cognitive neuroscience of social emotions and implications for psychopathology: examining embarrassment, guilt, envy, and schadenfreude. Psychiatry and Clinical Neurosciences, 68(5), 319-336. doi:https://doi.org/10. $1111 / \mathrm{pcn} .12182$

Kissler, J., Herbert, C., Winkler, I., \& Junghofer, M. (2009). Emotion and attention in visual word processing: an ERP study. Biological Psychology, 80(1), 75-83. doi:https://doi.org/10.1016/j.biopsycho. 2008.03.004

Koch, A., Alves, H., Kruger, T., \& Unkelbach, C. (2016). A general valence asymmetry in similarity: Good is more alike than bad. Journal of Experimental Psychology. Learning, Memory, and Cognition, 42(8), 1171-1192. doi:https://doi.org/10.1037/ $\mathrm{xlm} 0000243$

Kuperberg, G. R., \& Jaeger, T. F. (2016). What do we mean by prediction in language comprehension? Lang Cogn Neurosci, 31(1), 32-59. doi:https://doi.org/10.1080/23273798.2015.1102299

Kutas, M., \& Federmeier, K. D. (2011). Thirty years and counting: finding meaning in the N400 component of the event-related brain potential (ERP). Annual Review of Psychology, 62, 621-647. doi: https://doi.org/10.1146/annurev.psych.093008.131123

Lamm, C., Rutgen, M., \& Wagner, I. C. (2019). Imaging empathy and prosocial emotions. Neuroscience Letters, 693, 49-53. doi:https:// doi.org/10.1016/j.neulet.2017.06.054

Langeslag, S. J. E., \& Sanchez, M. E. (2018). Down-regulation of love feelings after a romantic break-up: Self-report and electrophysiological data. Journal of Experimental Psychology. General, 147(5), 720-733. doi:https://doi.org/10.1037/xge0000360

Langeslag, S. J. E., \& van Strien, J. W. (2018). Cognitive reappraisal of snake and spider pictures: An event-related potentials study. International Journal of Psychophysiology, 130, 1-8. doi:https:// doi.org/10.1016/j.ijpsycho.2018.05.010

Leon-Cabrera, P., Flores, A., Rodriguez-Fornells, A., \& Moris, J. (2019). Ahead of time: Early sentence slow cortical modulations associated to semantic prediction. Neuroimage, 189, 192-201. doi:https://doi. org/10.1016/j.neuroimage.2019.01.005

Leon-Cabrera, P., Rodriguez-Fornells, A., \& Moris, J. (2017). Electrophysiological correlates of semantic anticipation during speech comprehension. Neuropsychologia, 99, 326-334. doi: https://doi.org/10.1016/j.neuropsychologia.2017.02.026

Leuthold, H., Kunkel, A., Mackenzie, I. G., \& Filik, R. (2015). Online processing of moral transgressions: ERP evidence for spontaneous evaluation. Social Cognitive and Affective Neuroscience, 10(8), 1021-1029. doi:https://doi.org/10.1093/scan/nsu151

Li, J., \& Tomasello, M. (2018). The development of intention-based sociomoral judgment and distribution behavior from a third-party stance. Journal of Experimental Child Psychology, 167, 78-92. doi: https://doi.org/10.1016/j.jecp.2017.09.021

Malti, T., Gummerum, M., Ongley, S., Chaparro, M., Nola, M., \& Bae, N. Y. (2016). "Who is worthy of my generosity?" Recipient characteristics and the development of children's sharing. International Journal of Behavioral Development, 40(1), 31-40. Retrieved from $<$ Go to ISI $>$ ://WOS:000366603500004

McClure, S. M., York, M. K., \& Montague, P. R. (2004). The neural substrates of reward processing in humans: the modern role of FMRI. Neuroscientist, 10(3), 260-268. doi:https://doi.org/10.1177/ 1073858404263526

Melis, A. P., \& Semmann, D. (2010). How is human cooperation different? Philosophical Transactions of the Royal Society of London. Series B, Biological Sciences, 365(1553), 2663-2674. doi:https:// doi.org/10.1098/rstb.2010.0157

Mendes, N., Steinbeis, N., Bueno-Guerra, N., Call, J., \& Singer, T. (2018). Preschool children and chimpanzees incur costs to watch punishment of antisocial others. Nature Human Behaviour, 2(1), 45-51. doi:https://doi.org/10.1038/s41562-017-0264-5

Metusalem, R., Kutas, M., Urbach, T. P., Hare, M., McRae, K., \& Elman, J. L. (2012). Generalized event knowledge activation during online sentence comprehension. Journal of Memory and Language, 66(4), 545-567. doi:https://doi.org/10.1016/j.jml.2012.01.001

Moreno, E. M., \& Vazquez, C. (2011). Will the glass be half full or half empty? Brain potentials and emotional expectations. Biological Psychology, 88(1), 131-140. doi:https://doi.org/10.1016/j. biopsycho.2011.07.003

Nieuwland, M. S., Politzer-Ahles, S., Heyselaar, E., Segaert, K., Darley, E., Kazanina, N.,... Huettig, F. (2018). Large-scale replication study reveals a limit on probabilistic prediction in language comprehension. Elife, 7. doi:https://doi.org/10.7554/eLife.33468

Oldfield, R. C. (1971). The assessment and analysis of handedness: The Edinburgh inventory. Neuropsychologia, 9(1), 97-113. doi:https:// doi.org/10.1016/0028-3932(71)90067-4

Paulus, F. M., Muller-Pinzler, L., Stolz, D. S., Mayer, A. V., Rademacher, L., \& Krach, S. (2018). Laugh or cringe? Common and distinct processes of reward-based schadenfreude and empathy-based fremdscham. Neuropsychologia, 116(Pt A), 52-60. doi:https://doi. org/10.1016/j.neuropsychologia.2017.05.030

Peterburs, J., Voegler, R., Liepelt, R., Schulze, A., Wilhelm, S., Ocklenburg, S., \& Straube, T. (2017). Processing of fair and unfair offers in the ultimatum game under social observation. Scientific Reports, 7, 44062. doi:https://doi.org/10.1038/srep44062

Rigoni, D., Polezzi, D., Rumiati, R., Guarino, R., \& Sartori, G. (2010). When people matter more than money: an ERPs study. Brain Research Bulletin, 81(4-5), 445-452. doi:https://doi.org/10.1016/j. brainresbull.2009.12.003

Santamaria-Garcia, H., Baez, S., Reyes, P., Santamaria-Garcia, J. A., Santacruz-Escudero, J. M., Matallana, D., ... Ibanez, A. (2017). A lesion model of envy and Schadenfreude: legal, deservingness and moral dimensions as revealed by neurodegeneration. Brain. doi: https://doi.org/10.1093/brain/awx269

Schacht, A., \& Vrticka, P. (2018). Spatiotemporal pattern of appraising social and emotional relevance: Evidence from event-related brain 
potentials. Cognitive, Affective, \& Behavioral Neuroscience, 18(6), 1172-1187. doi:https://doi.org/10.3758/s13415-018-0629-x

Schindler, S., Schettino, A., \& Pourtois, G. (2018). Electrophysiological correlates of the interplay between low-level visual features and emotional content during word reading. Scientific Reports, 8(1), 12228. doi:https://doi.org/10.1038/s41598-018-30701-5

Schmidt, M., Rakoczy, H. (2019). On the uniqueness of human normative attitudes. . In K. Bayertz, Roughley N. (Ed.), The normative animal? On the anthropological significance of social, moral and linguistic norms. Oxford: Oxford University Press.

Sebastián-Gallés, N., Martí, M.A., Carreiras, M., \& Cuetos F. (2000). Lexesp: Una base de datos informatizada del español. Barcelona, Spain: Edicions de la Universitat de Barcelona.

Shamay-Tsoory, S. G., Ahronberg-Kirschenbaum, D., \& BaumingerZviely, N. (2014). There is no joy like malicious joy: schadenfreude in young children. PLoS One, 9(7), e100233. doi:https://doi.org/10. 1371/journal.pone. 0100233

Silk, J. B., \& House, B. R. (2016). The evolution of altruistic social preferences in human groups. Philosophical Transactions of the Royal Society of London. Series B, Biological Sciences, 371(1687), 20150097. doi:https://doi.org/10.1098/rstb.2015.0097

Singer, T., \& Klimecki, O. M. (2014). Empathy and compassion. Current Biology, 24(18), R875-R878. doi:https://doi.org/10.1016/j.cub. 2014.06.054

Takahashi, H., Kato, M., Matsuura, M., Mobbs, D., Suhara, T., \& Okubo, Y. (2009). When your gain is my pain and your pain is my gain: neural correlates of envy and schadenfreude. Science, 323(5916), 937-939. doi:https://doi.org/10.1126/science.1165604

Tomasello, M. (2019). Becoming Human: A Theory of Ontogeny. Cambridge, Massachusetts: Harvard University Press.
Urbach, T. P., \& Kutas, M. (2018). Cognitive Electrophysiology of Language. In S. A. R. M. G. Gaskell (Ed.), Oxford Handbook of Psycholinguistics (2nd ed.): Oxford University Press.

Van Berkum, J. J., Holleman, B., Nieuwland, M., Otten, M., \& Murre, J. (2009). Right or wrong? The brain's fast response to morally objectionable statements. Psychological Science, 20(9), 1092-1099. doi: https://doi.org/10.1111/j.1467-9280.2009.02411.x

Van Berkum, J. J., van den Brink, D., Tesink, C. M., Kos, M., \& Hagoort, P. (2008). The neural integration of speaker and message. Journal of Cognitive Neuroscience, 20(4), 580-591. doi:https://doi.org/10. 1162/jocn.2008.20054

Wicha, N. Y., Moreno, E. M., \& Kutas, M. (2004). Anticipating words and their gender: an event-related brain potential study of semantic integration, gender expectancy, and gender agreement in Spanish sentence reading. Journal of Cognitive Neuroscience, 16(7), 12721288. doi:https://doi.org/10.1162/0898929041920487

Yu, H., Nan, W., Yang, G., Li, Q., Wu, H., \& Liu, X. (2019). Your Performance Is My Concern: A Perspective-Taking Competition Task Affects ERPs to Opponent's Outcomes. Frontiers in Neuroscience, 13, 1162. doi:https://doi.org/10.3389/fnins.2019. 01162

Zhong, J., Liu, Y., Zhang, E., Luo, J., \& Chen, J. (2013). Individuals' attentional bias toward an envied target's name: an event-related potential study. Neuroscience Letters, 550, 109-114. doi:https:// doi.org/10.1016/j.neulet.2013.06.047

Publisher's note Springer Nature remains neutral with regard to jurisdictional claims in published maps and institutional affiliations. 